\title{
Morphological Analysis of Terminal Air Spaces by Means of Micro-CT and Confocal Microscopy and Simulation within a Functional Model of Lung
}

\author{
Andres Kriete $^{1}$, Henrik Watz ${ }^{2}$, Wigbert Rau $^{2}$, Hans-Rainer Duncker ${ }^{1}$ \\ ${ }^{1}$ Institute of Anatomy and Cell Biology \\ Image Processing Laboratory \\ Aulweg 123, 35385 Giessen, Germany \\ ${ }^{2}$ Department of Diagnostic Radiology \\ University Clinic Giessen \\ Klinikstr. 36, 35385 Giessen, Germany
}

\begin{abstract}
We devise a new methodology to investigate terminal air spaces of lung. Micro-CT imaging and a computer guided technique for scanning and image fusion in confocal microscopy enable the acquisition of extended and highly resolved data volumes exhibiting respiratory units. Segmentation and the analysis of the topography of respiratory airways leads to a finite volume model suitable for computational physics within a computer lung model.
\end{abstract}

\section{Introduction}

Terminal air spaces such as alveoli, alveolar ducts and acini, also called respiratory units, are responsible for the gas exchange and herewith represent the important functional part of lung. There are up to 150000 acini or respiratory units in mammalian lungs, each containing 300-2000 alveoli. The fundamental knowledge about the spatial and functional relationships of terminal air spaces need to be obtained before a structural and functional model can be constructed suitable for a computer simulation.

There is a long history of experimental difficulties in the quantitative investigation of terminal air spaces. Due to the lack of appropiate imaging techniques most studies were based on casts,e.g. [1], which do not permit any insight into the structural organization of the dense and undifferentiated lung tissue (see Fig. 1). This paper explores a new set of methodologies to image, quantify and model terminal air spaces of mammalian species including micro-CT and a complementary confocal microscopic imaging technique. 


\section{Material and Methods}

\subsection{Micro-CT}

Micro-CT investigations require structures of high density to give sufficient contrast. Applications reported so far are limited to the analysis of bones or applications in material sciences [2]. To give highly resolved images at sufficient contrast of the lung parenchyma, we introduce histological staining techniques not very much unlike to those known in light and electron microscopy. Exstirpated human lungs were inflated and fixed with hot formalin vapor via the main bronchus. Respiratory movements were simulated over a period of 8 hours to get a sufficient amount of fresh formalin vapor into the alveoli, which were inflated to nearly full inspiratory volume. Cylindric specimens of the fixed lungs of $10 \mathrm{~mm}$ in diameter were taken perpendicular to the lung surface. In order to augment the contrast between alveolar walls and air, the lung tissue was impregnated with 0.8 molar $\mathrm{AgNO}_{3}$. Subsequently the specimens were mounted in the micro-CT. An X-ray tube with a microfocus of 10 microns diameter was used as a source, a CCD-array as a detector, the peak of the X-ray spectrum was at $25 \mathrm{keV}$. The images acquired by this device have a spatial isotropic resolution of 14 microns at a matrix of $1024^{2}$ pixels in an isotropic volume data set.

Visualization of lung parenchyma is a difficult task. Because of an undifferentiated appearance, no prominent structures can be outlined. Visualizations of surfaces without preprocessing don't give any insight, but deliver a sponge like appearence. An interesting feature to visualize the lumen is that of virtual endoscopy, as illustrated in Fig. 2. However, for a morphological analysis a carefull segmentation technique, such as the one described in Chapter 3, has to be applied.

\subsection{Confocal microscopy}

The confocal microscope is used in the sense of an optical tomograph to acquire image sequences with precise registration at thick specimen. The axial resolution of such an device is improved with lenses of high numerical aperture, but unfortunatly the today available lens designs bring along a narrow field of view at the same time. Certain studies like that of the lung parenchyma however require both a good axial resolution and in particular a wide field of view. This conflict is resolved here by an image fusion technique which combines a series of histological thick sections, each of these sections is confocally resolved and scanned in an array-like fashion and all the resulting subvolumes are digitally combined. The distinct advantage of using this 


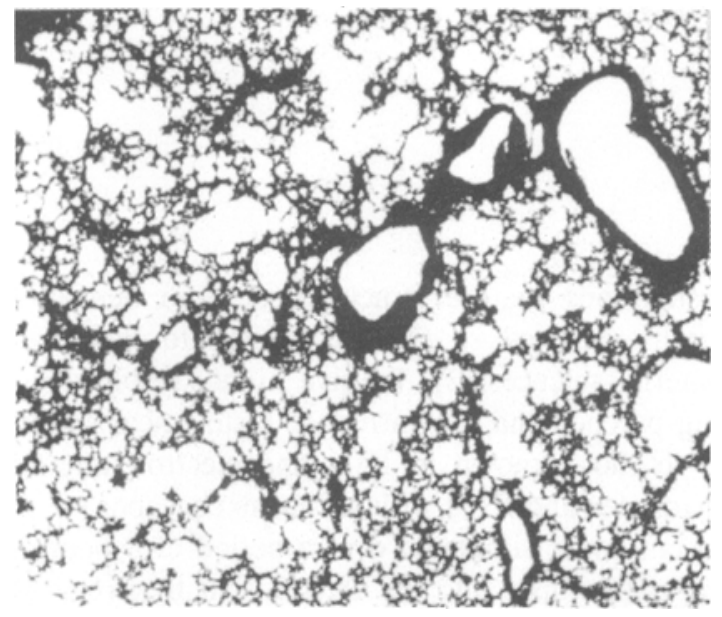

Fig.1 A section of lung tissue (rat) imaged with a confocal microscope. This image illustrates the undifferentiated appearence of the densily packed structures, only some prominent bronchioli can be easily identified. Size is about $2 \times 2 \mathrm{~mm}$

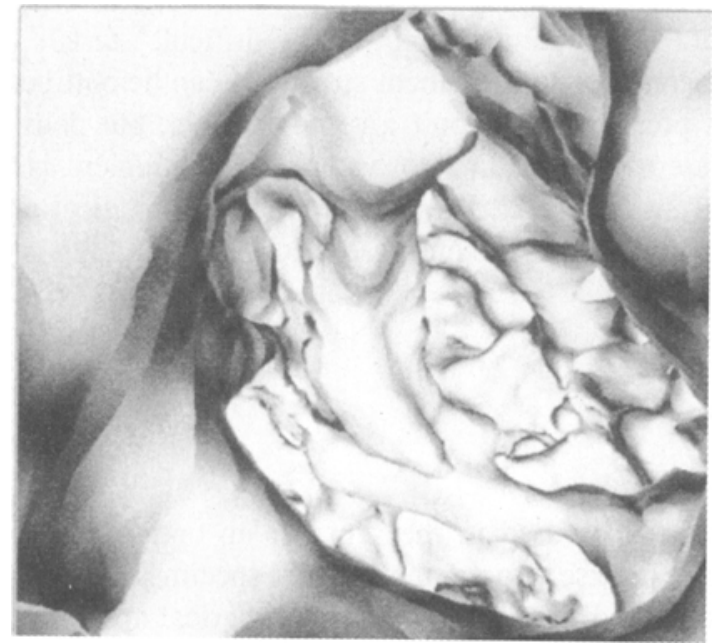

Fig.2 Endoscopic view of the acinar pathways at a human respiratory unit. The data set stems from micro-CT imaging, size of the pathway is about $0.05 \mathrm{~mm}$ in diameter 
imaging technique is that it is scalable for resolution and is also usefull for other than human lungs when higher resolutions are required, such as rat or mouse.

Histological sections of 50-80 micron thickness of a rat lung stained with $\mathrm{HE}$ are the material for this example. For imaging, an upright Zeiss confocal laser scan microscope was used (Zeiss LSM 410) equipped with a HeNe laser emitting at 535 $\mathrm{nm}$. Fluorescence was detected with one photomultiplier above $545 \mathrm{~nm}$ from the lung parenchyma.

To increase the accessible volume in confocal microscopic imaging, a framework for a computer guided imaging technique was developed. First the sections were imaged with a low magnifying lens of $5 \times$ (imaged area was $2000 \times 2000$ microns). These images were aligned for linear shift and rotation. Transfered back to the confocal microscope, these preprocessed images serve for alignement of the histological sections. Variation of the scanning offset, i.e. shifting the scanning beam electronically away from the center in all $\mathbf{8}$ possible directions at a lateral distance of 600 microns, gave 9 reference images and at a zoom factor of 4 they construct a frame of $1800 \mu \mathrm{m}$ side length (see Fig. 1). Switching to a 20x magnifying lens, the field of view at zoom factor 1 fits exactly to that of the 9 individual reference images, so that non-overlapping subvolumes can be acquired by optical sectioning. Typically, some hundred images have to be fused, the example given in Fig. 3 is based on 819 fused images of $768 \times 768 \times 91$ voxels each, equivalent to a volume of $1800 \times 1800 \times 910$ microns. Such a volume may enclose a complete acinus, but exhibits as well all structural details like the alveoli.

\section{Segmentation and 3-D Analysis of Terminal Air Spaces}

The segmentation and labeling of structures within lung parenchyma is a step to prepare measurements and structural attributes for 3-D visualizations. Segmentation often requires interaction by the user, since automatic algorithms almost rely on the intensities of the voxels only. Here, the threshold selected was interactively controlled, which is used as a demarcation between lung parenchyma and background.

Once binary images had been obtained, it was necessary to identify structures out of the images like the outer boundary of the acinus, ductus, bronchi and alveoli. During the labeling the black and white pixel are replaced by user selected gray levels. To differentiate ductus and alveolar sacs completely from the alveoli, binary morphological operations using structuring elements for erosion and dilatation were performed. This procedure, if performed several times, removed all structures lying beyond a given size. Here, the filter size and the number of operations were selected in a way that mostly all alveoli, many of them in a sponge like open form, could be differentiated from the ducts. By this procedure, about $80 \%$ of all structural details 
were identified. Since the exact boundary of the acinus is difficult to define, in particular in the outer areas some of the structures remain questionable and had to be identified interactively.

The overwhelming structural detail of the data sets make the above described labeling method very time-consuming, since all the procedures have to be applied anew for the sections following until the whole data set is processed. What was tested instead was a still more automated seeding procedure. First, one representative image out of the middle of the data stack was selected as a starting point. At this image, an interactive guided labeling of structures as described above was performed. Next, this identified image was used as an marker or reference for the following one. If combined with one selected label of the reference image, this label was transferred to those objects which overlap by at least one pixel of the area with the reference object. Due to changes from section to section not all the structures were identified correctly, and some interactive correction was always necessary. Fig. 3 depicts an completely segmented data set with opaque rendered bronchioli. Alveolar ducts and aveoli have a high transparency.

For a morphological analysis of the branching pattern of airways within an acinus, a topological skeleton was extracted. We used our software package 3D-TOP, explained in detail elsewhere [3]. This skeleton may be embedded in a volume rendering, such as in Figure 3. The topological skeleton starts with the bronchiolus terminalis, splits into two short segments, which are devided up further into two daughter ducts each. Assuming a circular form of the ducts, the cross-sectional area was calculated. The sum of cross-sectional areas along the distance from the origin of the acinus reveals a normal, Gaussian distribution. The maximum of the distribution occurs around the 5-6 th order of branching.

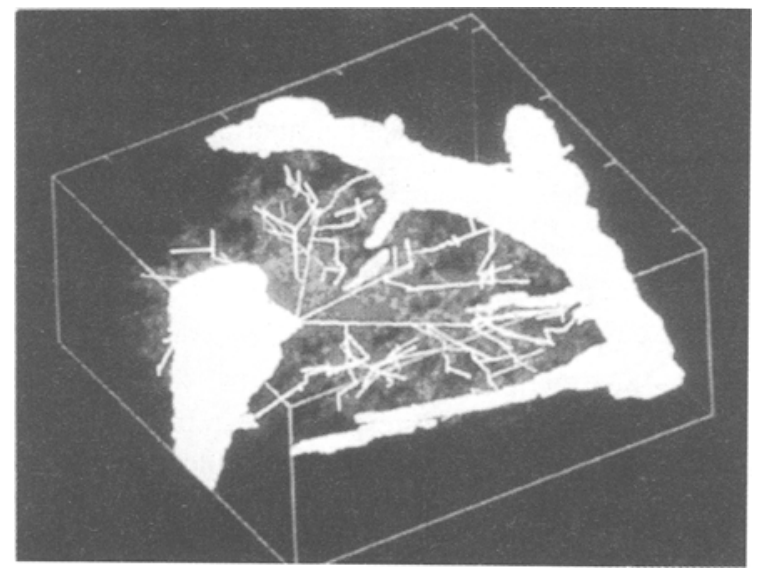

Fig.3 A volume rendered view of a complete respiratory unit with bright bronchioli. Embedded lines indicate the branching pattern of the airspaces. Size is about $2 \times 2 \times 1 \mathrm{~mm}$ 


\section{Simulation of Gas Transport within a Computer Lung Model}

A computer modeling is often complicated by the structural complexity at the various levels [4]. As an example, the mammalian lung requires an investigation of some thousand air conducting bronchial segments and, in addition, the investigation of respiratory units (acini) at microscopic resolution as discussed here. The use of models for substructures is essential to realize a computer aided functional simulation of complex biological systems like that of a lung. As an initial step, a hierarchical model was reported previously which concerns the structure of the bronchial, air conducting part of the rat lung. This model features about 4000 bronchial segments $[5,6]$, to which a dynamical model of the respiratory units has to be attached.

The Gaussian shape of the diameter distribution of airways at respiratory units allows to develop a model being composed of individual volumetric segments or subsections. This procedure is a typical approach in computational fluid dynamics [7]. Based on the analytical findings reported above, a Gaussian volume distribution can be modeled consisting of individual segments. From here the number of alveoli and the volume-to-surface ratio can be calculated. Depending on the breathing amplitude typical variations in volume size of all segments within the acini occur (Fig. 4).

To model gas distribution, each segment gains mass from the distal segment or losses mass due to transport into the daughter segments or gas uptake. Parameters necessary to perform such calculations include diffusion coefficient, difference of concentration across segment and gradient, way of flow partitioning and dispersion coefficient $[6,8]$.

For each of the segments, such an equation describing conservation of mass can be postulated. A solution of the complete bronchial tree can not be found analytically, instead an iterative method was used, where the breathing cycle is devided up into many time-steps. The number of steps necessary depends on the mathematics used to yield a stable solution, typically 10000 time steps are necessary. For each of the segments at each time step the mass transport equation which is a differential equation is solved by Gauss-Seidel iteration. The calculation proceeds from the first order branches to more distal branches down to the respiratory units, and the resulting concentrations are stored along with the segments. The actual concentration values may be visualized.

Calculations can be performed for various amplitudes of the breathing cycle within the complete model of the organ of a rat lung. It was found that mass transport is about equal for convection and diffusion when entering the acinus, but the fast decrease in flow velocity quickly drops the rate of convectional mass transport. Diffusion however, dependent on the cross-sectional areas of the 
individual segments, increases towards the center of the acinus. Diffusional mass transport drops at a higher rate towards the periphery of respiratory units because of oxygen absorption.

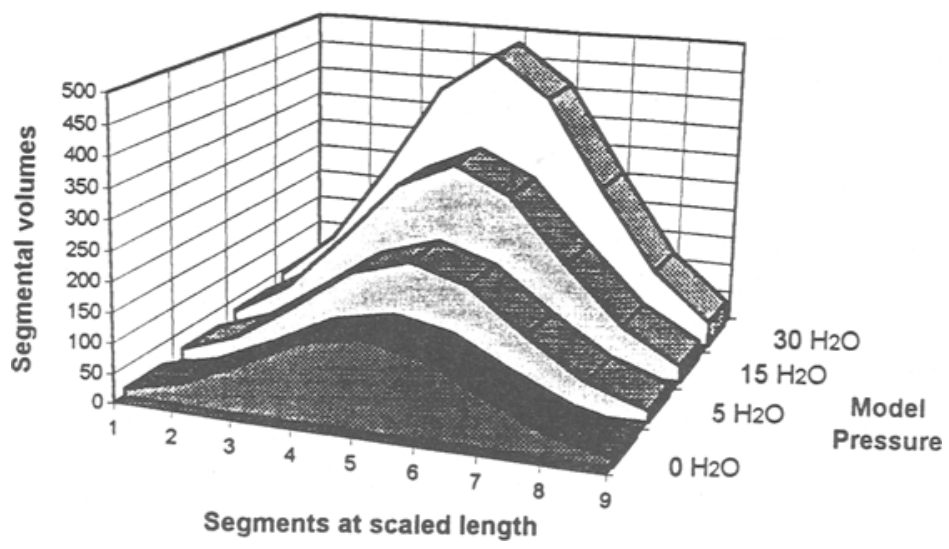

Fig. 4. Variation of segment sizes of modeled acini at different tidal volumes

\section{Conclusions}

For the investigation of terminal air spaces at the respiratory part of lung two novel imaging techniques are explored, which close a gap between the field of view obtainable with high resolution clinical imaging devices and the resolution of microscopical imaging. Complementary to micro-CT of stained lungs a novel 3-D image compositing technique at precise registration is described, which increases the accessible volume imaged with a confocal laser scanning microscope. The latter method is scalable and can also be applied to smaller mammalien lungs. Both methods allow to image complete respiratory units with all structural details.

The problems involved in the segmentation and visualization of the undifferentiated lung parenchyma is discussed, as well as the topological quantification of the acinar pathways. Subsequently a model of respiratory units is 
developed based on finite volumes to be used within a functional model of lung incorporating dynamics at breathing. Mass transport equations are solved iteratively describing convection, diffusion and gas uptake. Depending on the size and location of these units, a different amount of gas concentration and uptake is predicted .

Research addressing the microstructure of lung can now benefit of digital image processing by using advanced imaging techniques. Overall, new roads are opened to develop precise functional computer lung models usefull in clinical investigations, research and teaching.

\section{Acknowledgements}

We would like to thank K-P.Valerius, Giessen for loaning corrosion casts and histological sections. Special thanks go to T.Hildebrand and P.Rügsegger from the ETH Zürich for support in micro-CT imaging.

\section{References}

[1]Haefeli-Bleuer,B; Weibel,E.R.(1988): Morphometry of the humal pulmonary acinus. Anat.Record, 220, pp. 401-414

[2]Ruggsegger,P.,Koller, B., Müller, R.(1996) A microtomographic system for the nondestructive evaluation of bone architecture. Calcif.Tissue Res., 58, pp 24-29

[3]Kriete,A., Schwebel,T.(1996) : 3-D Top - a software package for the topological analysis of image sequences. J. of Structural Biology 116 , Academic Press (1996), pp 150-154

[4]Hersh,J.S.(1990): A survey of modeling representations and their application to biomedical visualization and simulation. in: Proc. of the Conf. on Visualization in Biomedical Computing. Atlanta, IEEE Comp.Soc.Press, pp 432-440

[5]Kriete,A.(1969): Hierarchical data representation of lung to model morphology and function. in: (Eds. Höhne,K.-H., Kikinis, R).: Visualization in Biomedical Computing. Springer, NY, pp 399-404

[6]Kriete,A.(1998): Form and function of mammalian lung: analysis by scientific computing. in press for: Advances in Anatomy, Embryology and Cell Biology, Vol. 145, Springer Verlag, Berlin

[7]Davidson,L., Nielsen,P.(1995): Calculation of two-dimensional airflow in facial regions and nasal cavities using an unstructured finite volume solver. Conference on Finite Volume Methods, ISSN 1395-7953 R9539, Dec.1995

[8]Mercer,R.R.,Anjilvel,S.,Miller,F.J.,Crapo,J.D.(1991): Inhomogenity of ventilatory unit volume and its effects on reactive gas uptake. J.Appl.Physiol. 70,5, pp 2193-220 